\title{
Formación de los recursos humanos en Estadística. Cuba, 1959-2008
}

\author{
Formation of human resources in Statistics in Cuba from 1959 \\ to 2008
}

\begin{abstract}
Ileana Elena Castañeda Abascal ${ }^{\mathrm{I}}$; Liana Margarita Labrada Moreno ${ }^{\mathrm{II}}$; Juliette Masip Nicot ${ }^{\mathrm{II}}$; Eudis Nápoles Wilson ${ }^{\mathrm{II}}$; Nuria Delis Despaigne ${ }^{\mathrm{II}}$; Meralys Moreno Gelis ${ }^{\mathrm{II}}$; Gustavo Sánchez Fernández ${ }^{\mathrm{II}}$
\end{abstract}

IDoctora en Ciencias de la Salud. Bioestadística de II Grado. Escuela Nacional de Salud Pública. La Habana, Cuba.

${ }^{\mathrm{II}}$ Residente de 3er. año en Bioestadísticas. Escuela Nacional de Salud Pública. La Habana, Cuba.

\section{RESUMEN}

La humanidad ha necesitado contar con información estadística desde su surgimiento. En Cuba se constata importantes registros, sobre todo a partir del siglo XIX. Después del triunfo de la Revolución, la recolección, análisis y publicación de las estadísticas sanitarias se consideró una tarea prioritaria encaminada al mejoramiento de la gestión y la investigación de salud. Este estudio muestra las diferentes etapas por las que transitó la formación de recursos humanos en estadística en el nivel técnico y profesional y la inclusión de los conocimientos de esta ciencia en los programas de las carreras de ciencias médicas en el período de 1959 al 2008. Se enfatiza el contexto histórico y los factores que estuvieron presentes en cada etapa del periodo estudiado. Desde 1961 se comenzó la formación de Auxiliares de Estadística, que se capacitaron hasta alcanzar el segundo nivel, posteriormente se comenzó la formación de Técnicos de Estadística de Salud todo lo cual permitió cubrir la estructura estadística que alcanzó cobertura nacional; en la actualidad la formación técnica puede culminar en la Licenciatura de Gerencia en Información en Salud. Desde los primeros años de la Revolución se 
comenzó a impartir en las carreras de medicina y estomatología contenidos de estadística. El desarrollo científico-técnico alcanzado en el país hizo que en 1970 comenzara la formación de un profesional dedicado a la bioestadística, para lo que se creó una residencia cuyo plan de estudio se ha perfeccionado en tres momentos con la finalidad de incrementar la calidad y cantidad de los conocimientos impartidos.

Palabras clave: Formación de recursos humanos, estadísticas, bioestadísticas, Cuba.

\begin{abstract}
Mankind has required statistical information since its origin. It has been confirmed that important records existed in Cuba since the $19^{\text {th }}$ century. After the triumph of the revolution, the health statistics collection, analysis and dissemination were considered a priority task aimed at the improvement of health management and research. This study showed the different stages of the formation of human resources in statistics at the technical and professional levels, and the inclusion of pieces of knowledge of this science in the medical science curricula in the 19592008 period. The historical context and the factors involved in each stage of the studied period were emphasized. From 1961 on, the formation of statistics assistants started, then they were trained to reach the second level; later the formation of health statistics technicians began, all of which allowed covering the statistical structure nationwide. At present, the technical education may finish with a degree in Health Information Management. Since the first years of the Revolution, statistics was included as subject in medical and dentistry studies. The scientific and technical development of the country made it possible that in 1970, the formation of professionals in biostatistics started, for which a residency was approved and its curricula has been upgraded at three different moments in order to raise the quality and range of knowledge and learning.
\end{abstract}

Key words: Formation of human resources, statistics, biostatistics, Cuba.

\title{
INTRODUCCIÓN
}

Desde los comienzos de la civilización, los hombres necesitaron cuantificar el número de personas, animales o ciertas cosas de su vida cotidiana. Se han encontrado evidencias de los registros de información que desarrollaron civilizaciones como la Egipcia, la Griega, la China y el Imperio Romano que florecieron en el periodo antes de nuestra era. En los libros bíblicos se puede encontrar los datos de la población de Israel y del bienestar material de las diversas tribus judías. ${ }^{1}$

Los esfuerzos en este sentido continuaron a lo largo de la historia, ejemplos elocuentes son el censo realizado en Inglaterra en 1086. En ese mismo país se comenzó a realizar el primer registro de nacimientos en el siglo XIV y en 1662 
apareció el primer estudio estadístico notable de población, realizado por John Graunt. $^{1}$

En la ciudad de Breslau, en Alemania, se realizó en 1691 un estudio sobre mortalidad que fue utilizado por el astrónomo inglés Edmund Halley como base para la primera tabla de mortalidad. En 1706, Francois Bossier de Lacroix (Savages) realizó el primer intento de clasificación de las causas de muerte que se lograron concretar con los aportes de William Cullen, y se sometieron a revisión cada 10 años. $^{2}$

En el siglo XIX, con la generalización del método científico para estudiar todos los fenómenos de las ciencias naturales y sociales, los investigadores aceptaron la necesidad de utilizar la información numérica. Se destacaron en este siglo los estudios de series de mortalidad realizados por William Farr en $1839 .^{2}$

En 1939, el ilustre profesor Raymond Pearl comenzó a impartir clases en la Universidad "John Hopkins" en la asignatura de Biometría y Estadísticas, lo que sin duda marca el comienzo de la formación de recursos humanos en esta área de la salud pública. En el siglo xx, el desarrollo de la computación permite un avance ostensible en los procederes estadísticos complejos.

Los orígenes de las estadísticas en Cuba se remontan a la labor desempeñada por médicos, quienes además de realizar sus funciones asistenciales, se ocupaban de recoger, analizar y publicar las estadísticas. En 1833, Don Ramón de la Sagra estudió las Tablas Necrológicas Mortuorias del Cólera de La Habana y sus arrabales según sexo, raza, edad, condición social y nacionalidad. En el año 1842, en reunión celebrada por la Junta Superior de Sanidad, sus miembros expresaron: "Es necesario trabajar en la recolección de la información estadística de mortalidad y sus causas, para poder planificar acciones de salud a partir de los datos obtenidos."3

En el año 1845, se imprime el primer libro dedicado a las estadísticas médicas titulado "Ensayo estadístico de la mortalidad en la diócesis de la Habana. Año 1843", realizado por el doctor Ángel J. Cowley. Los datos recogidos clasifican a los fallecidos por sexo, raza, lugar de residencia y causas, estas últimas agrupadas en 75 rubros ordenados alfabéticamente. ${ }^{3}$

Durante el último cuarto del siglo XIX, se destacó en esta rama de las ciencias médicas el doctor Ambrosio González del Valle y Cañizo, que publicó 18 años consecutivos sus "Tablas Obituarias", con la mortalidad de La Habana y principales ciudades del país. ${ }^{3}$

A fines del mencionado siglo, comienza sus estudios estadísticos el doctor Jorge Le Roy y Cassá, quien laboró por más de 40 años en esta ciencia, dejando a su muerte, una valiosa colección de trabajos, que han servido como referencias para dar continuidad a las series de estadísticas médicas del país y que le valieron el título de Padre de las Estadísticas Sanitaria y Demográfica de Cuba. ${ }^{3}$

En 1936, el Dr. Rafael Calvo puso en vigor el modelo de recogida de información administrativa y asistencial para hospitales y en 1942, el Dr. Jorge A. Bustamente recogió y analizó información sobre enfermedades mentales. Otras publicaciones que hicieron aportes científicos de interés en ese período fueron: "La Mortinatalidad y Mortalidad Infantil en Cuba" del Dr. Rafael Fosalba y "Taller de Vida de Ciudad de la Habana" del Dr. Rafael Jorge Vega Lomar. ${ }^{3}$ 
A partir de la primera mitad de la década de los 20 , con la destitución del Secretario de Salubridad y Beneficencia Juan Guiteras Gener, por el presidente Alfredo Sayas, a petición de Enoch $\mathrm{H}$. Crowder, representante del gobierno de los Estados Unidos en Cuba, se produce un deterioro de las estadísticas sanitarias en el país, tan es así que en 1959 se publicó el último número del Boletín de Sanidad y Asistencia Social con información de $1953 .^{3}$

Con el triunfo de la Revolución, el gobierno revolucionario consideró como una tarea prioritaria, el mejoramiento de la salud del pueblo cubano y las funciones relacionadas con las estadísticas sanitarias tenían sin duda un papel importante en este empeño. Es así que en agosto de 1961 se estipula por la ley número 965, también conocida como Ley Orgánica del Ministerio de Salud Pública, que entre las funciones del nuevo ministerio estaba la de organizar las estadísticas. Para lograr este propósito se hizo necesario la formación de personal capacitado para desempeñar esta tarea en todos los niveles del Sistema de Salud y las regiones del país. ${ }^{4}$

La formación de recursos humanos en estadística aplicada a las ciencias médicas y la salud pública ha evolucionado desde 1959 en correspondencia del encargo social que se ha hecho a las escuelas y a la universidad. El desarrollo alcanzado ha contribuido al mejoramiento de la gestión y de las investigaciones en salud.

El presente estudio tiene el objetivo de mostrar las diferentes etapas por las que transitó la formación de recursos humanos en estadística a nivel técnico y profesional, así como la inclusión de los conocimientos de esta ciencia en los programas de las carreras de ciencias médicas en el período de 1959 al 2008. Se hace énfasis en el contexto histórico y los factores que estuvieron presentes en cada etapa del periodo estudiado.

\section{MÉTODOS}

Se realizó una investigación descriptiva de corte histórico.

\section{Fuentes de información}

\section{Documentales}

Programas de la Especialidad de Bioestadística en sus cuatro ediciones.

Programas de las carreras de Ciencias Médicas.

Registro de Profesionales del MINSAP

\section{Testimoniales}

Se realizaron entrevista en profundidad a personalidades que fueron protagonistas en la formación de recursos humanos en estadística dentro de la salud en determinados momentos del periodo estudiado.

Las características de los procesos de formación en estadística se lograron mediante la aplicación del método de análisis y síntesis a partir de las revisiones bibliográficas. 
La información recogida en las entrevistas en profundidad se procesó con la utilización del análisis de contenido que permitió arribar a las categorías más importantes.

Con todo lo anterior se aplicó el método histórico que permitió ordenar los acontecimientos.

\section{EVOLUCIÓN HISTÓRICA DE LA FORMACIÓN DE RECURSOS HUMANOS EN ESTADÍSTICAS EN EL SISTEMA NACIONAL DE SALUD}

La estrategia cubana para la creación y consolidación del sistema de salud se ha favorecido en gran medida por la política de formación de recursos humanos en el sector. Se ha desarrollado un riguroso programa de capacitación de profesionales y técnicos para el sector de la salud. Las instituciones formadoras tanto universitarias como técnicas, se encuentran adscritas directamente al MINSAP, bajo la rectoría metodológica de los ministerios de Educación y Educación Superior. El número de instituciones docentes se ha incrementado ostensiblemente a lo largo de la etapa. Sólo se contaba con una escuela de medicina en 1959 mientras en la actualidad existen 21 facultades de ciencias médicas, cuatro institutos superiores de ciencias médicas, cuatro facultades de estomatología y cuatro facultades de enfermería que han graduado 91571 médicos, 13342 estomatólogos y 34146 licenciados en enfermería. A esta cifra se debe adicionar los 592 graduados que desde el 1994 ha aportado la Licenciatura de Tecnología de la Salud. La cifra de centros dedicados a la formación técnica en salud varió de uno a 57, lo que coadyuvó a que en el año 2006 se encontraran trabajando 2591 técnicos de estadística. ${ }^{2,5,6}$

\section{FORMACIÓN DE TÉCNICOS DE ESTADÍSTICAS DE SALUD}

En los primeros años de Gobierno Revolucionario se comienza a ampliar la cobertura de los servicios de salud con la creación de numerosas instituciones sobre todo en las provincias del interior del país. La organización descentralizada de la función estadística en el sector demandaba de un personal capacitado para el procesamiento de la información estadística que se hacía más numeroso y complejo. Esta es la razón por la que en 1961 se comienza a impartir el Curso de Auxiliar de Estadística en la Escuela Sanitaria del Instituto "Finlay", la cual fue fundada por el Decreto Presidencial $N^{\circ} .1521$ el 16 de enero de 1927 . El curso estaba dirigido por la doctora Carmen Pascual Jacomino, quien había sido formada como Máster en Administración en Salud en la década de los 50 en México. ${ }^{7}$

La Escuela Sanitaria del Instituto "Finlay" fue la sede del Curso de Auxiliar de Estadística por haber sido una institución de prestigio histórico en la formación de los recursos humanos relacionados con la salud pública. En sus inicios se impartieron clases teórico-prácticas a los jefes locales de sanidad, funcionarios de la Secretaría de Salud, damas isabelinas, enfermeras y obreros de la Junta Local de Sanidad de La Habana. Posteriormente se diversificaron los contenidos docentes impartidos y el tipo de personal que entrenaba. En 1952 se agregaron nuevos cursos de especialización para técnicos de laboratorio, técnicos de rayos $X$, parasitología y administradores de hospitales. La promulgación de la Ley $\mathrm{N}^{\circ}$. 607, aparecida en la Gaceta Oficial el 21 de octubre de 1959, reguló el funcionamiento de la carrera sanitarista y se reactivó la Escuela Sanitaria en el antiguo local del 
Instituto "Finlay", la cual constituía una Dirección General del Ministerio de Salubridad y Asistencia Social. Por Decreto Presidencial del 24 de mayo de 1963 la escuela cambió su nombre a Escuela de Salud Pública de La Habana. ${ }^{8,9}$

Para el ingreso en el curso de Auxiliar de Estadística era necesario tener nivel medio superior, se exigía el título de bachiller o contador. En la primera convocatoria se contó con 22 matriculados provenientes de las seis provincias existentes en esos momentos, cuatro de la región sur de Oriente, cuatro de Camagüey, tres de Holguín, tres de Villa Clara, una de Matanzas, dos de Pinar del Río y cinco de La Habana. El primer curso tuvo una duración de ocho semanas en las que se impartieron conocimientos de Estadística Descriptiva, Higiene, Epidemiología, Matemática, Educación para la Salud. Posteriormente se ofreció un curso de dos semanas de duración sobre codificación aplicando la Séptima Clasificación Internacional de Enfermedades que contó con la asesoría de un profesor chileno. El curso de codificación fue recibido también por las codificadoras que ya trabajaban en el Ministerio de Salud Pública.

En ese mismo momento se desarrolló otro curso en el hospital "Comandante Manuel Fajardo" donde se impartieron contenidos que no estaban incluidos en el anterior, tales como Admisión, Archivo y Estadística Hospitalaria (Álvarez Dedien Y, ex-jefe de Estadística del Hospital Infantil Sur de Santiago de Cuba. Comunicación personal,2008I).

Con posterioridad los conocimientos de ambos cursos se fundirían en el de Auxiliar de Estadística.

En las ediciones posteriores del curso de Auxiliar de Estadística fue necesario disminuir los requisitos de ingreso por no existir suficientes personas con nivel medio superior. Por consiguiente se aumentó el tiempo de formación a seis meses. Para estos cursos se capacitaron a un grupo de profesores como Mario Quiroz Vento que se preparó en México y el Dr. Álvaro Díaz Artilde que estudió en Brasil. Se incluyeron los contenidos de Admisión, Archivo y Estadística Hospitalaria que no se abordaron en la primera edición. De esta forma se realizaron seis cursos en La Habana con carácter nacional, el último fue en 1965. Se impartía más de un curso por año, ya que era necesario cubrir las necesidades de auxiliares de estadística en las provincias. Se había logrado que en el año 1965 ya existieran 80 auxiliares de estadística formadas en esta escuela, cifra que se elevó a 381 en 1964. Eso posibilitó cubrir los cargos a nivel de provincia, en las unidades hospitalarias más complejas y otros niveles territoriales intermedios de todo el país (Dra.C. Eneida Ríos Massabot. Funcionaria del MINSAP en esos momentos. Comunicación personal, 2008).

En 1964 se impartió el curso de Segundo Nivel dirigido al perfeccionamiento de los Auxiliares de Estadística. Además, algunos de ellos se capacitaron en otros países como Chile. De esta forma se contó con un capital humano que podía desarrollar funciones docentes, lo que conformó el claustro de profesores que se necesitaba para la creación de las Escuelas de Auxiliares de Estadística en cada una de las provincias del país. Villa Clara, Camagüey, Santiago de Cuba, Pinar del Río fueron las primeras provincias en fundar sus escuelas y posteriormente se sumaron Holguín y Matanzas. La Escuela de Salud Pública de La Habana continuó siendo la sede de los cursos de Segundo Nivel. Los planes de estudio del curso de Auxiliar de Estadística y el de Segundo Nivel mantuvieron un continuo desarrollo lo que permitió el enriquecimiento de cada edición con nuevos conocimientos.

En 1965 se trasladó la Escuela de Salud Pública al local del antiguo hospital de la Policía Nacional bajo la dirección del doctor Carlos Martínez Reyes (1922-1983), 
quien era el profesor jefe del departamento de Administración de Salud Pública. En este nuevo local es que se van a iniciar las residencias de Higiene, Epidemiología, Administración de Salud y posteriormente la de Bioestadística. ${ }^{9}$

En los años 70 se introduce en Cuba el concepto de Técnico Medio. En este momento a todos los egresados con los títulos de Auxiliar de Estadística y Segundo Nivel se les convalida y se les otorga el título de Técnico Medio de Estadística. Se crea, bajo la dirección del Dr. Francisco Rojas Ochoa, Máster en Administración de Salud, el curso de Técnico Medio de Estadística. El plan de estudio tenía una duración de tres años. En el primer año se impartían las asignaturas que contenía el curso de Auxiliar de Estadística, el segundo año estaba destinado a prácticas docentes y el tercero incluía las asignaturas del Segundo Nivel.

A mediados de la década de los 80 se suspende la formación de los Técnicos de Estadística de Salud. Los puestos de trabajo fueron ocupados por los graduados como Técnicos de Estadística Económica que no contaban con la suficiente formación en el área de la salud. Se demandó un esfuerzo adicional de las personas e instituciones con el fin de preparar convenientemente a dichos técnicos para que se pudieran desempeñar convenientemente.

En el año 2003 se comienza la formación de los Técnicos de Estadísticas dentro del programa de formación de la licenciatura de Gerencia en Información en Salud. En este presente año 2008 se graduó el primer grupo de licenciados de esta rama.

Es importante destacar que la formación de los Codificadores del país ha sido independiente de la de los Técnicos. Reciben un conocimiento básico de estadística y se les imparte un curso de perfeccionamiento especial para desempeñar su labor.

Desde los primeros años de la Revolución se pudo contar con el personal técnico necesario para cubrir las plazas existentes en todos los niveles de la estructura estadística del país. El desempeño de los técnicos ha permitido la sostenibilidad de los registros de información estadística en el sector de la salud. La preparación ha pasado por varias etapas en consonancia con la necesidad del perfeccionamiento de la formación.

\section{ESPECIALIDAD EN BIOESTADÍSTICA}

En el año 1970 el Dr. Francisco Rojas Ochoa quien se encargaba de la dirección del departamento de Estadísticas del Ministerio de Salud Pública, se percata de que no era suficiente la calidad e integridad de las estadísticas continuas que se estaba logrando con el concurso de los Técnicos de Estadística. Durante los años 1967 y 1968 se habían creado los Institutos de Investigación de Nefrología y Cardiología, entre otros, que demandaban de profesionales dedicados a la estadística, es decir bioestadísticos, para cumplir la misión investigativa que rebasaba la asistencia médica. Se gestiona becas para médicos y algunos técnicos de experiencia a través de la OMS para cursar entrenamientos de bioestadística a las Escuelas de Salud Pública de Chile, Inglaterra y en las Universidades de Columbia y Michigan, lo cual fue un paso importante para contar con el capital humano capaz de resolver los problemas con la aplicación del método científico.

Años antes el Dr. Arnaldo Tejeiro Fernández recibió formación en estadística durante un curso sobre Malaria en Venezuela y al Dr. Francisco Rojas Ochoa se le confirió una beca en Francia. Los dos profesionales mencionados anteriormente, 
junto con el entonces técnico Cándido López Pardo, quien se graduó con honores de Máster en la Universidad de Columbia, en Estados Unidos de América, diseñaron un curso de adiestramiento en Bioestadística. Se instó a los directores de los institutos de investigación a que enviaran a recibir este curso a un médico representante de cada institución. Se pretendía que con este adiestramiento los graduados pudieran solucionar las tareas de investigación del instituto, sin dejar de ejercer sus funciones en la especialidad de base (Dr.C. Francisco Rojas Ochoa, quien fue uno de los que concibieron el curso de Bioestadística para los médicos. Comunicación personal, 2008).

Se trató de captar a licenciados en Matemática para que ejercieran en el campo de la estadística en el sector de la salud, el escaso el número de graduados de la Universidad de La Habana y la gran demanda por parte de otros ministerios impidió contar con la cantidad que se pretendía. No obstante, es importante destacar que se cuenta en Cuba con prestigiosos matemáticos especializados en la Bioestadística. Algunos llegaron egresados de la carrera y fueron asignados a la Dirección Nacional de Estadísticas, como el hoy Dr.Cs. Luis Carlos Silva. Otros se insertaron en cuarto año de la carrera para hacer sus prácticas docentes y se vincularon a la especialidad y al grupo de investigación dirigido por el Dr. Arnaldo Tejeiro. Su aporte a la especialidad ha sido valioso, llegaron incluso, a formar parte del claustro de profesores. Entre ellos se pueden mencionar a la Dra.C. Mercedes Rubén Quesada, Lic. Carlos Sánchez Texidó y Dr.C. Jorge Bacallao Gallestey.

A inicios de la década del 70 existen las condiciones creadas para aplicar lo dispuesto en la Reforma de 1962 que reglamenta las especialidades en las distintas ramas de la medicina. ${ }^{8}$ Es así que en 1970 comienza la formación de la primera especialista en Bioestadística que fue una mujer, la Dra. María Amparo Pascual. No existían antecedentes de la enseñanza de la especialidad en Cuba, por lo que se hizo una exhaustiva investigación de los programas utilizados en escuelas de salud pública de otros países. Se recibió la valiosa colaboración de asesores de la OPS, entre ellos se destaca la del matemático John Fertig, Profesor Titular de la Escuela de Salud Pública de la Universidad de Columbia, New York, el profesor José Coll, médico argentino graduado como Máster en Estadística en la Escuela de Higiene y Medicina Tropical de Londres bajo la tutela del prestigioso estadístico Sir Austin Bradford Hill (1897-1991), Harvey Goldtein quien tuvo un notable aporte a la formación de la Dra.C. Mercedes Rubén Quesada en el campo de las investigaciones sobre crecimiento y desarrollo. Otras personalidades que apoyaron la consolidación de la Bioestadística fueron John Biby y RR. Puffer, esta última reconoció internacionalmente la calidad de las Estadísticas de Salud Cubanas y participó en los procesos de formación de esta disciplina en el país (Dr.C. Francisco Rojas Ochoa, creador de la especialidad. Comunicación personal, 2008).

La primera edición de la especialidad se desarrolló mediante cursos afines a la estadística que se impartían en distintas instituciones del país, entre ellos se encontraban los cursos de Matemática y Filosofía que se impartían en el Centro Nacional de Investigaciones Científicas (CENIC); y el curso de Ruta Crítica de la Universidad de La Habana, entre otros. El primer año se hizo en forma conjunta con las especialidades de Higiene, Epidemiología y Administración de Salud y su sede estaba en la Escuela Nacional de Salud Pública, los otros dos años se cursaban en la sede de la Dirección Nacional de Estadísticas del MINSAP. La Dra.C. Eneida Ríos Massabot impartía los conocimientos de estadísticas sanitarias y tutoraba a los residentes en las actividades afines con las estadísticas continuas; mientras el Dr. Arnaldo Tejeiro Fernández tutoraba las que se relacionaban con la investigación. Con esta organización se editaron dos cursos más de residentes. Los requisitos para la matricula era ser graduado de medicina o estomatologíaa (Eneida Ríos Massabot, creadora de la especialidad. Comunicación personal, 2008). 
En el año 1974 es cuando se inicia la formación en Bioestadística con un programa de estudio totalmente estructurado. Contemplaba un primer año común con las otras especialidades de la salud pública, el segundo año se hacía en la Dirección Nacional de Estadísticas del MINSAP, que era la institución encargada de coordinar con los profesores cubanos y extranjeros propios de la rama de la Estadística encargados de impartir los contenidos (Dra.C. Rosa Jiménez Paneque y Dra.C. Leticia Fernández Garrote, alumnas del curso. Comunicación personal, 2008).

Por Resolución Ministerial $\mathrm{N}^{\circ} 55$ A del 6 de septiembre de $1976{ }^{10}$ se creó el Instituto de Desarrollo de la Salud (IDS), inaugurado el 6 de septiembre de 1977 en el local del antiguo hospital antituberculoso "Doctor Joaquín García Lebredo", municipio de Arroyo Naranjo, bajo la dirección del Dr.C. Francisco Rojas Ochoa, Profesor de Mérito del Instituto Superior de Ciencias Médicas de La Habana, a esta institución se integró la Escuela de Salud Pública, por lo que se trasladan al IDS las residencias de Higiene, y Epidemiología. Bioestadística situó su sede en este Instituto, ya que asumió las funciones de investigación en salud que se realizaban en la Dirección Nacional de Estadísticas. ${ }^{3}$

Las residencias que funcionaban en el IDS tenían sus propios programas de estudio, sin embargo, existían asignaturas que eran comunes para varias especialidades, por ejemplo: Análisis Matemático se impartía en Bioestadística y Administración de Salud y Estadística Descriptiva en Bioestadística y Epidemiología. ${ }^{11}$

La experiencia de la especialidad alcanzada en Cuba implicó que se realizaran ajustes al plan de estudios hasta que se hizo evidente la necesidad de revisarlo íntegramente y reformularlo. En el año 1981, se contó con la aprobación de la Dirección Docente de Posgrado del MINSAP para la elaboración de un nuevo plan de estudio. Se creó una comisión encargada de acometer el encargo constituida por expertos en Bioestadística, siendo la primera vez que participaran un número importante de graduados de la propia especialidad. La primera tarea consistió en la elaboración de una definición precisa sobre la disciplina, con el fin de identificar su objeto de estudio, su cuerpo de conocimientos, lo que serviría de sustento teórico para trazar los objetivos y contenidos del plan de estudios, así como los aspectos que condicionaban la estrategia docente. ${ }^{12}$

El objeto de estudio de la Bioestadística se definió como: el conocer el estado de salud de la población y constituir un medio para elevar la calidad de la gestión en salud. Su sistema de conocimientos debe sintetizarse en los principios teóricos procedentes de las ciencias médicas y otras ciencias sociales integrados unitariamente con los medios matemáticos y estadísticos en el estudio de los fenómenos salud-enfermedad. ${ }^{12}$

De esa forma quedó diseñado el plan de estudio de 1982 que estuvo estructurado por un Modelo del Especialista que incluía a su vez tres modelos: de formación comunista, de calificación ocupacional y de formación profesional. Este último incluía las siguientes funciones: de servicio, de estadísticas de salud, investigativa, docente y administrativas; tenía una duración de tres años académicos, distribuidos en seis semestres y estaba organizado por asignaturas y actividades de servicio, docentes e investigativas.

La relevancia ganada por la especialidad desde su surgimiento hasta esta fecha se demostró en el Taller Nacional de Investigación en Salud celebrado en 1983 donde 17 de los 68 asistentes eran especialistas en Bioestadística, profesionales dedicados a la especialidad. ${ }^{13}$ 
Por Resolución Ministerial N 192 de 1987 fue creada la Facultad de Salud Pública adscripta al Instituto Superior de Ciencias Médicas de La Habana a la que se incorporaron las funciones docentes y de investigación del IDS. ${ }^{10}$ Es por esta razón que en el curso 1986-1987 las residencias relacionadas con la salud pública dentro de las cuales se encuentra la Bioestadística continuaron realizándose en esa institución.

La evolución lógica de la ciencia en general, así como el de la salud pública y la computación en particular influyeron en el desarrollo de la especialidad y por tanto en la necesidad de rediseñar el plan de estudio de la residencia que fue aprobado en el año 1993. Este plan se organizó por módulos que respondían a las cuatro Funciones de Salida de los egresados: servicios de estadística, investigación, docencia y dirección. El tiempo de duración era de tres años, en el primero se hizo énfasis en los contenidos relacionados con la salud pública, sobre todo los de Dirección, Higiene y Epidemiología, además los módulos básicos de la especialidad como el Análisis Matemático y el Algebra; en el segundo año estaban contempladas la mayoría de las técnicas estadísticas, dentro de las que se agregaron las que demandan para su procesamiento del uso de la informática como el Análisis Multivariado, Análisis de Supervivencia y Series Cronológicas; en el tercer año como otra novedad de este plan, se incorporaron módulos relacionados con el diseño de investigación: Investigación en Sistemas y Servicios de Salud, Investigación Epidemiológica e Investigación Clínica. ${ }^{14}$

La ampliación y profundización de los contenidos de Higiene y Epidemiología y Dirección incorporados al plan de estudio aprobado en 1993 se consideró un paso de avance en la formación de un especialista más integrado al quehacer de la salud. No obstante, con la práctica se constató la necesidad de reducir contenidos sobre todo los de Higiene de acuerdo a las necesidades específicas de la especialidad.

Se decidió rediseñar nuevamente el plan de estudio para solucionar definitivamente el problema antes mencionado y responder a las demandas siempre crecientes de la ciencia. Para este fin, se realizó una investigación que se denominó "Desarrollo de la Especialidad en Bioestadística" que exploró la situación internacional de la disciplina en cuanto a programas docentes existentes y líneas de investigación. Dentro del país se indagó sobre los aportes de la especialidad a la salud pública así como los principales problemas que demandan el concurso de los bioestadísticos.

En el mes de julio del 2000 se nombró al Doctor Eugenio R. Borroto Cruz como último Decano de la Facultad de Salud Pública y poco después se llevó a cabo el traslado para el edificio situado en las esquinas de las calles Línea e I, en el Vedado, municipio Plaza de la Revolución. La Facultad de Salud Pública se funde con el Centro Nacional de Perfeccionamiento Médico y de Medios de Enseñanza (CENAPEM) que estaba enclavado en dicho edificio por la Resolución $\mathrm{N}^{\circ}$ 246/03 del Ministerio de Economía y Planificación, de 28 de abril de 2003, de esta forma surge oficialmente la Escuela Nacional de Salud Pública (ENSAP). En institución tuvo la responsabilidad de formar especialistas en Bioestadística.

En el curso 2005-2006, el Ministerio de Salud Pública, mediante la Resolución No. 246, aprobada el 27 de septiembre del 2006, orienta la reducción del tiempo de duración de las especialidades médicas a dos años. Con este fin se realizó el último rediseño del plan de estudio de la especialidad de Bioestadística bajo la dirección del Viceministerio de Docencia e Investigaciones del Ministerio de Salud Pública, y la Escuela Nacional de Salud Pública. 
En este plan, vigente en la actualidad, se mantiene la estructura por Funciones. Se unificaron algunos módulos y se organizaron teniendo en cuenta siete áreas temáticas: Computación y Gestión de la Información, Fundamentos de la Salud Pública, Estadísticas de Salud y Población, Bases para la Especialización en Bioestadística, La Investigación en Salud, Enseñanza de la Bioestadística y Técnicas Estadísticas.

Desde 2006 se pasó la sede de la especialidad de Bioestadística al Instituto Nacional de Higiene, Epidemiología y Microbiología.

Hasta ese momento la especialidad ha mantenido un carácter nacional. Sin embargo, en el 2008 se comenzó a trabajar intensamente en la descentralización hacia las facultades e institutos de ciencias médicas del país. Cuando se consolide este empeño se podrá formar un número mayor de especialistas.

La cantidad de médicos y estomatólogos dedicados y/o especializados en Bioestadística ha tenido una tendencia ascendente, en los primeros años de la década del 80 del pasado siglo el promedio se encontraba alrededor de los 30 profesionales, mientras que desde comienzos del presente siglo el Sistema Nacional de Salud cuenta con más de 200 dedicados o especializados en esta disciplina. La profesión médica ha sido la más representada.

Desde sus inicios las mujeres se vincularon activamente al desarrollo de la especialidad, no se puede dejar de mencionar a la Dra.C. Eneida Ríos Massabot, a quien se considera junto al Dr. Francisco Rojas Ochoa y al Dr. Arnaldo Tejeiro Fernández fundadores de la especialidad. La primera graduada como se ha dicho fue una mujer, y esta tradición se mantuvo con el decursar del tiempo.

Los primeros especialistas en Bioestadística se desempeñaron fundamentalmente en los institutos de investigación y en la Universidad. Posteriormente cubrieron las solicitudes hechas por los diferentes niveles de la estructura estadística, se tiene la experiencia de trabajos reconocidos en las provincias e incluso en los municipios. En los departamentos responsabilizados con la investigación de las instituciones de salud ha existido siempre una representación importante de especialistas en Bioestadística. La demanda creciente de ese profesional en las instituciones de salud se debe a que el aporte de las funciones que pueden desarrollar es reconocido con fuerza por los directivos de salud.

\section{BIOESTADÍSTICA EN LOS PROGRAMAS DE LAS CARRERAS DE CIENCIAS MÉDICAS}

Con el triunfo revolucionario del 1ro. de enero de 1959 se crean las condiciones históricas favorables para llevar a feliz término los ideales reformistas en las antiguas facultades de Medicina y Odontología de la Universidad de La Habana. Este proceso de reforma de estudios, que conmocionó principalmente a la Facultad de Medicina, se extendió hasta 1962 y se caracterizó por una intensa lucha política enmarcada en el reinicio de las actividades docentes y el apoyo universitario al gobierno revolucionario. ${ }^{15}$

El 10 de enero de 1962, aniversario 33 de la muerte de Julio Antonio Mella, se proclamó la Ley de Reforma de la Enseñanza Superior en la escalinata de la Universidad de La Habana y tomó posesión de su cargo el nuevo Rector, el Doctor Juan Marinello Vidaurreta. ${ }^{5}$ A partir de este momento, los médicos y estomatólogos 
pudieron contar con la formación social y humanística que requería el proyecto social de la Revolución Cubana. ${ }^{16}$

La Reforma amparó la creación de un nuevo plan de estudio, que por primera vez incluyó asignaturas como Materialismo Histórico, Materialismo Dialéctico, Economía Política y Metodología Estadística, entre otras. Es este, el primer plan de estudio de las carreras de Medicina y Estomatología que incluía conocimientos referentes al manejo estadístico de la información en salud pública.

En 1962 se crea el departamento de Metodología Estadística y se imparte por primera vez la asignatura, pero al año siguiente, en 1963, debido al éxodo masivo de médicos hacia el exterior, y al descenso consiguiente del número de galenos en la isla, se hace necesario poner en práctica el método conocido como "Plan Baeza" en el cual, sin perder de vista los objetivos generales del anterior, se adecua la carrera a una extensión de cinco cursos. Se mantuvo la asignatura de Metodología Estadística pero se fusionó con la de Epidemiología. ${ }^{17}$

En 1966 se pone en vigor un plan de estudio muy similar al de 1962, que separa nuevamente las asignaturas de Metodología Estadística y Epidemiología. La primera se comenzó a impartir en el segundo año de la carrera con una frecuencia de seis $h$ semanales, durante seis semanas. El incipiente desarrollo que se había alcanzado en la formación de recursos humanos en estadística influyó notablemente en los resultados satisfactorios que tuvo la implementación de la asignatura. El claustro de profesores estuvo dirigido por el Dr. Arnaldo Tejeiro Fernández en la Facultad de Santiago de Cuba y por el Dr. Francisco Rojas Ochoa en Ciudad de La Habana, en ambos casos se contó con el apoyo de los Auxiliares de Estadísticas graduados [Delgado García G. Planes de estudio. Capítulo de la Investigación "Historia de la Enseñanza Superior de la medicina en Cuba (1900-1962)". Inédita, en proceso de publicación. Copia en Archivo de la Oficina del Historiador del MINSAP].

La asignatura de Estadística ha formado parte de los planes de estudio de las carreras de Medicina y Estomatología de forma ininterrumpida, se incluyó por tanto en los planes de estudio de 1978, de 1986 y en el de 2005; en este último se fusionó con Computación por entenderse que es una disciplina que apoya y acompaña el trabajo de la Estadística, a esa asignatura de le denominó Informática Médica y Bioestadística que se imparte en el segundo año de las carreras.

A pesar de la temprana incorporación de los contenidos de estadística en los planes de estudio de Medicina y Estomatología y su permanencia a lo largo de los casi 50 años de Revolución, aún persisten dificultades en la formación. Es posible que esto se deba, por una parte, al momento de la carrera en que se imparte la asignatura y por otra, la necesidad de retomar los conocimientos adquiridos sistemáticamente y en forma práctica.

\section{CONSIDERACIONES FINALES}

La formación de personal técnico de estadística ha estado relacionada con la demanda de los servicios de salud desde el triunfo de la Revolución. Las etapas más importantes aportan la formación de Auxiliares de Estadística, que se capacitaron hasta alcanzar el Segundo Nivel, posteriormente comenzó la formación de Técnicos de Estadística de Salud y en la actualidad se forman en Licenciatura de Gerencia en Información en Salud. 
La especialidad de Bioestadística surgió en Cuba en el año 1970 y se consolidó como residencia para los egresados de pregrado de Medicina y Estomatología en 1974. Su plan de estudio original creado en este año ha sido renovado en tres ocasiones: en 1982, en 1993 y finalmente en el 2005, en cada una de ellas se ha incrementado la calidad y cantidad de los conocimientos impartidos en función del desarrollo de la ciencia y la demanda social.

Los contenidos de Estadística se incorporaron a las carreras de Medicina y Estomatología desde los primeros años de la Revolución y se han mantenido hasta el momento actual por considerarse que aportan al desarrollo humanístico de los graduados y los dota de herramientas importantes para el trabajo en las disciplinas de la salud pública y en la investigación.

\section{REFERENCIAS BIBLIOGRÁFICAS}

1. Valdés F. Comprensión y aplicación de la Estadística. Universidad Rómulo Gallego. [actualizado, citado 12 Jun 2008]. Disponible en:

http://www.cortland.edu/flteach/stats/stat-sp.html

2. Swaroop S. Estadísticas Sanitarias. La Habana: Ediciones Revolucionarias;1964.

3. López Serrano E. Desarrollo histórico de las estadísticas sanitarias en Cuba. Selección de Artículos Estadísticos. La Habana: Facultad de Salud Pública;1987.

4. Ley 965. Ley Orgánica del Ministerio de Salud Pública. La Habana: MINSAP; 1961. [Revisada en el archivo personal del Dr. Francisco Rojas Ochoa].

5. Rojas Ochoa F. Situación, sistema y recursos humanos en salud para el desarrollo en Cuba. Rev Cubana Salud Pública. 2003;29(2):157-69.

6. MINSAP. Anuario Estadístico de Salud 2006. Palcograf. La Habana: MINSAP;2007.

7. López Serrano E. Instituto Finlay. Primera Escuela de Administradores de Salud en Cuba. Rev Cubana Adm Salud. 1986;12 (3):285-9.

8. Delgado García G. La formación de médicos salubristas en Cuba: algunos aspectos históricos. Cuad Hist Salud Pública [serie en Internet]. 2006 June [citado 13 Jun 2008]; (99). Disponible en:

http://scielo.sld.cu/scielo.php?script=sci arttext\&pid=S0045 -

$91782006000100002 \& \operatorname{lng}=$ en\&nrm $=$ iso

9. Delgado García G. Precursores y Forjadores de la Salud Pública Cubana. Dr. Carlos M. Martínez Reyes (1922-1983). Rev Cubana Salud Pública. 1992;18(1):702.

10. Ministerio de Salud Pública. Resolución Ministerial № 192 de 1987. La Habana: MINSAP; 1987 . [Copia fotostática en Archivo de la Oficina del Historiador del Ministerio de Salud Pública].

11. Plan de Estudios para la especialidad de Bioestadística 1978. Programas. La Habana: MINSAP, Escuela Nacional de Salud Pública. [Revisado en Archivos de la Vicedirección Docente de la Escuela Nacional de Salud Pública]. 
12. Plan de Estudios para la especialidad de Bioestadística. Programas. La Habana: MINSAP, Instituto de Desarrollo de la Salud;1982.

13. Taller Nacional de Investigación en Salud. La Habana: MINSAP;1983.

14. Plan de Estudios para la especialidad de Bioestadística. Programas. La Habana: Facultad de Salud Pública, MINSAP;1992.

15. Teja Pérez J, Biosca Adán L, Delgado García G, Companioni Landín FA, Céspedes Carrillo A, Inclán López D, et al. La reforma universitaria de 1962 en la Facultad de Ciencias Médicas de la Universidad de La Habana. Rev Cubana Educ Med Super [serie en Internet]. 2004 May-Ago [citado 13 Jun 2008];18(3).

Disponible en: http://scielo.sld.cu/scielo.php?pid=S0864-

21412004000300008\&script=sciarttext

16. Delgado García G. El profesor Roberto Guerra Valdés y la reforma universitaria de 1962. Rev Cubana Educ Med Super [serie en Internet]. 2001 May-Ago [citado 13 Jun 2008]. Disponible en: http://scielo.sld.cu/scielo.php?pid=S0864$\underline{21412004000300008 \& \text { script }=\text { sci a }}$

17. Delgado García G. Conferencias de Historia de la administración de Salud Pública en Cuba. La Habana: MINSAP;1996.

Recibido: 9 de septiembre de 2008.

Aprobado: 18 de octubre de 2008.

Ileana Elena Castañeda Abascal. Escuela Nacional de Salud Pública. Calle Línea esq. I. El Vedado 10400. La Habana, Cuba. 